\title{
SIMPLIFIED UPPER POLE NEPHRECTOMY: INITIAL EXPERIENCE
}

\author{
UBIRAJARA BARROSO JR, ANTONIO J. VINHAES, MILTON S. BARROS, ADRIANO A. \\ CALADO, ANTONIO MACEDO JR, MIGUEL SROUGI
}

\begin{abstract}
Department of Urology, San Raphael Hospital and Federal University of Bahia, Salvador, Bahia, and Department of Urology, Paulista School of Medicine, Federal University of Sao Paulo, UNIFESP, Sao Paulo, SP, Brazil
\end{abstract}

\begin{abstract}
Objective: To assess the results of an upper pole nephrectomy technique on 5 children.

Materials and Methods: Upper pole nephrectomy was performed on 5 children, including 4 females and 1 male. Age ranged from 3 to 6 years old. The technique was performed without initial dissection of the renal pedicle. The upper pole is incised and removed. Upon its complete dissection, the segment that drains the upper pole is easily identified, clamped and sectioned.

Results: Three children with ureterocele and 2 with ectopic ureter underwent this procedure. There was no intra- or postoperative complication with this technique. DSMA scintigraphy showed no decrease in renal function in the remaining kidney following the procedure.

Conclusion: The polar nephrectomy technique is simple, and has the advantage of not approaching the renal hilum, which makes surgery less laborious and prevents risk of renal damage, hemorrhage and decreased function in the remaining renal portion.
\end{abstract}

Key words: kidney; ureter; children; abnormalities; ectopic; ureterocele

Int Braz J Urol. 2005; 31: 157-60

\section{INTRODUCTION}

Ureteropelvic duplication is the most frequent abnormality in the upper urinary tract (1). When the upper pole is not functioning, the standard treatment is nephrectomy of this segment (2). On children, this surgery is usually performed through a classic open approach, with isolation of the renal vascular pedicle, clamping of the upper segment of the renal artery and excision of the ischemic segment. However, arterial dissection increases surgical time, and isolation of the upper segment of the renal artery is sometimes not feasible, and there is an additional risk of vascular lesion (3). As well, the standard technique runs the risk of causing function loss in the lower pole in around $5 \%$ of cases because of damage to vessels irrigating this segment (3-6). Jednak et al. described a simplified upper pole nephrectomy technique where the dissection of the renal vascular pedicle is avoided (7). Thus, there is a reduction in operative time and surgical risk, in addition to maximizing the remaining renal function. However, there has been no other series reporting further experience with this technique, and we describe here our initial experience.

\section{MATERIALS AND METHODS}

Upper pole nephrectomy was performed on 5 children, including 4 females and one male. Age range was from 3 to 6 years old.

With the patient in a lumbotomy position, an incision is performed on the flank between the 11th and 12 th ribs. In contrast to Jednak et al. (7), who approach the kidney at the beginning of the proce- 
dure, we first isolate the ureter that drains the upper pole. In this stage, we perform a small incision in the ureter and introduce a $6 \mathrm{~F}$ urethral catheter towards the kidney. This makes identification of the ureteropelvic junction easier when the upper pole is approached. The catheter is fixed by means of non-absorbable suture. Next, we identify the upper pole, which presents a clear delimiting line with the lower pole. The polar dissection is facilitated by applying sutures that pull the upper pole. Following complete dissection, the pole in incised for its entire diameter towards the renal hilum. From this point, we identify the ureteropelvic orifice through the presence of the previously inserted catheter (Figure-1).

Following the incision in the upper pole, the base of the collecting system is incised in the same direction. Next, we section the segment at the limiting line between the 2 poles, taking care not to incise the collecting system at the lower kidney. During this stage, small bleeding can occur at the parenchymal edge, which can be easily identified and controlled with catgut suture.

Close to the renal hilum, we can easily identify vessels that irrigate and drain the upper pole. The segmental artery is easily isolated, clamped and sutured. The next stage consists of pulling the ureter by the site where it was approached in the early phase of surgery, and sectioning it at the lowest point possible (Figures-2 and 3). A schematic drawing for better understanding is present on Figure-4.

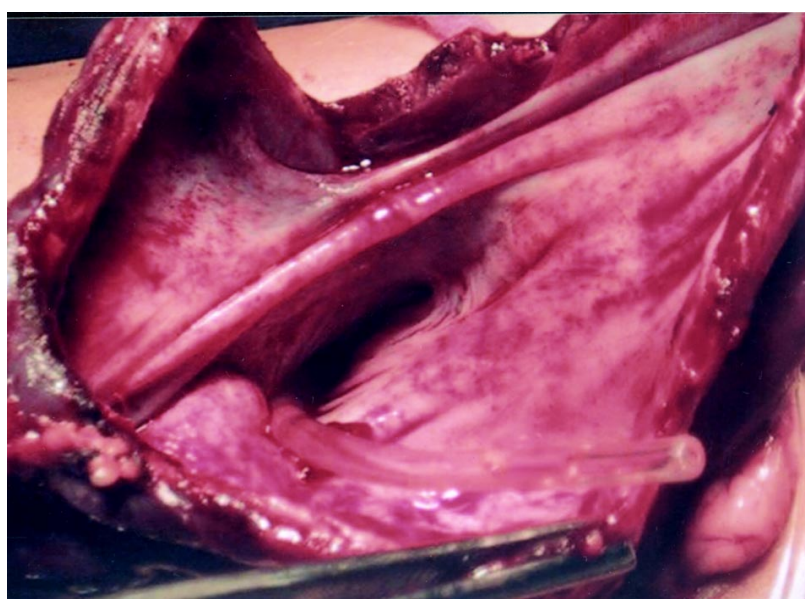

Figure 1 - Incised upper pole. Note the exteriorized catheter through ureteropelvic junction.

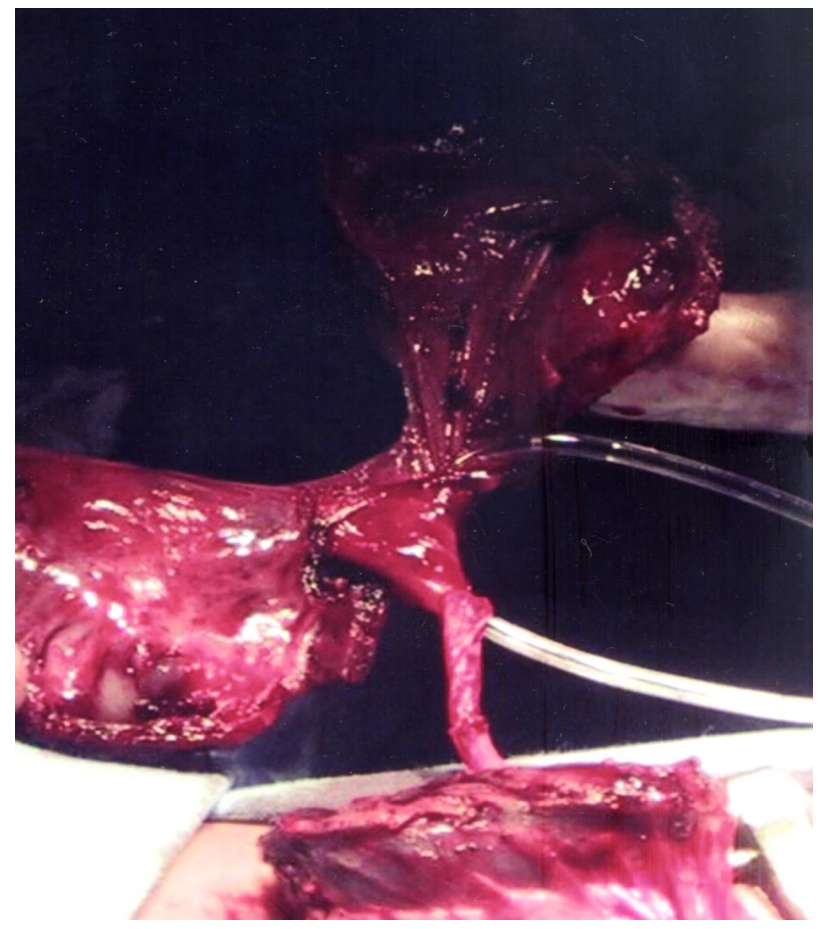

Figure 2 - The entire upper pole is dissected and incised in 2 portions. Note the catheter in the upper ureter close to the ureteropelvic junction.

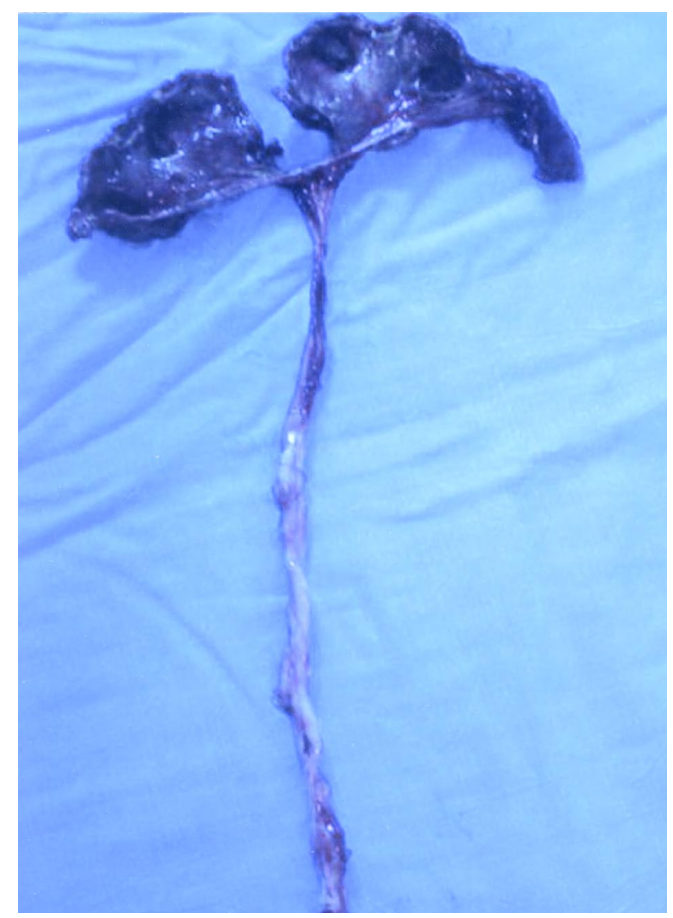

Figure 3 - Surgical specimen. Note the renal upper pole and corresponding ureter. 

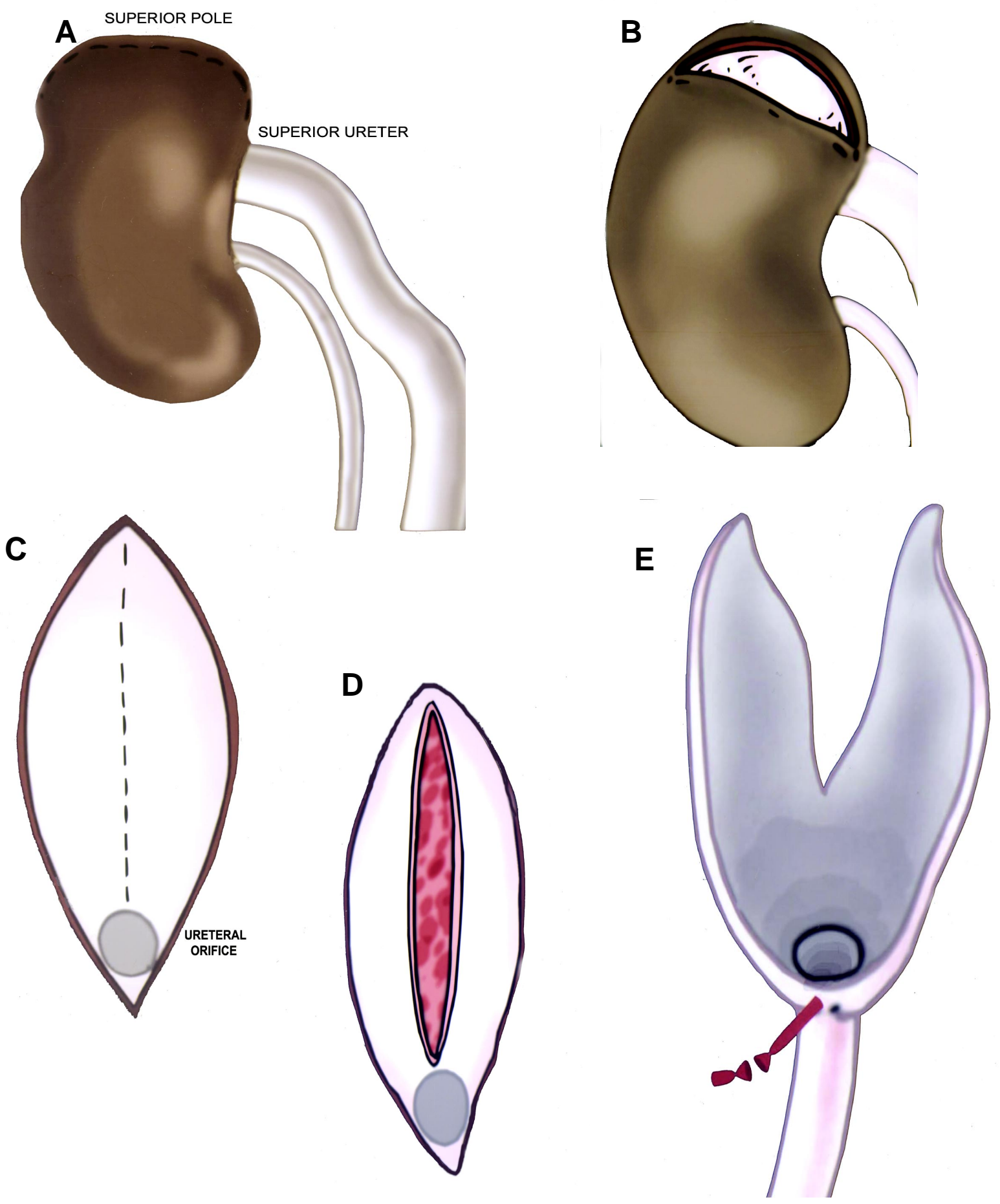

Figure 4 - A) The dotted line shows the incision site of the upper pole; B) The incised upper pole; C) Internal view of the upper pole, showing the incision site; D) Internal view of the upper pole, with the segment base previously incised (only the collecting system is incised); E) Dissection of the entire upper pole and ligation of the upper segment of the renal artery under direct view. 
The edges between the lower pole and the excised upper pole are then closed with tensionless 3-0 plain catgut sutures. If required, fat can be interposed between the stitches.

\section{RESULTS}

All patients had a non-functioning upper pole as shown by renal scintigraphy using dimercapto succinic acid (DMSA). Three patients had intravenous urographies showing good drainage of contrast medium only in the lower renal system. Of the 5 children, 3 had ectopic ureterocele and 2 had ectopic ureter.

There were no transoperative intercurrences in any case. No child was left with external drainage. Discharge from hospital occurred on the 1st postoperative day in one patient, on the 2 nd day in 3 patients and on the 3rd day in 1 patient. All patients underwent renal DMSA scintigraphy from the 2nd postoperative month on, with no case showing a decrease in renal function.

\section{COMMENTS}

We present our initial experience using a simplified polar nephrectomy technique (7). This technique is easy to perform and has the advantage of not requiring dissection of the renal hilum and facilitating the excision of the upper pole, which is performed under direct viewing. Non-dissection of the hilum prevents iatrogenic lesions, reduces surgical time and prevents the ligation of branches that irrigate the lower pole, and thus avoids decreased function in this segment (4-6). Additionally, vascular dissection is difficult in some cases. The excision of the upper pole is performed under direct

\section{Correspondence address:}

Dr. Ubirajara Barroso Jr.

Rua Alameda dos Antúrios, 212 / 602

Salvador, Bahia, 40280-620, Brazil

Fax: + $5571350-4722$

E-mail: ubarroso@uol.com.br viewing after the parenchyma is opened, minimizing the risks of inadvertent excision of the lower pole. This procedure accomplishes the goal of the surgery; that is, to maximally preserve the remaining renal function. With this technique, the segmental artery is easily identified and ligated under direct view far from the main renal artery.

In all our cases, surgery was successfully performed with no intra- or postoperative complications and with no loss of renal function on postoperative DMSA scintigraphy. In conclusion, the simplified technique for upper pole nephrectomy is a simple, fast and effective method for treating children with function loss in this segment.

\section{REFERENCES}

1. Schlussel RN, Retic AB: Ectopic Ureter, Ureterocele, and Other Anomalies of the Ureter. In Walsh PC, Retik AB, Vaughan ED, Wein AJ (eds.), Campbell's Urology. Philadelphia, W.B. Saunders. 1970; 8th ed., vol. 3, chap. 58, pp. 2007-52.

2. Ade-Ajayi N, Wilcox DT, Duffy PG, Ransley PG: Upper pole heminephrectomy: is complete ureterectomy necessary? BJU Int. 2001; 88: 77-9.

3. Spence HM: Nephro-ureterectomy and heminephroureterectomy in infancy and childhood. J Urol. 1954; 71: 171-82.

4. Belman AB, Filmer RB, King LR: Surgical management of duplication of the collecting system. J Urol. 1974; 112: 316-21.

5. Decter RM, Roth D, Gonzales ET: Individualized treatment of ureteroceles. J Urol. 1989; 142: 535-7.

6. Mor Y, Ramon J, Raviv G, Jonas P, Goldwasser B: A 20-year experience with treatment of ectopic ureteroceles. J Urol. 1992; 147: 1592-4.

7. Jednak R, Kryger JV, Barthold JS, Gonzalez R: A simplified technique of upper pole heminephrectomy for duplex kidney. J Urol. 2000; 164: 1326-8.

Received: September 23, 2004 Accepted after revision: March 11, 2005 